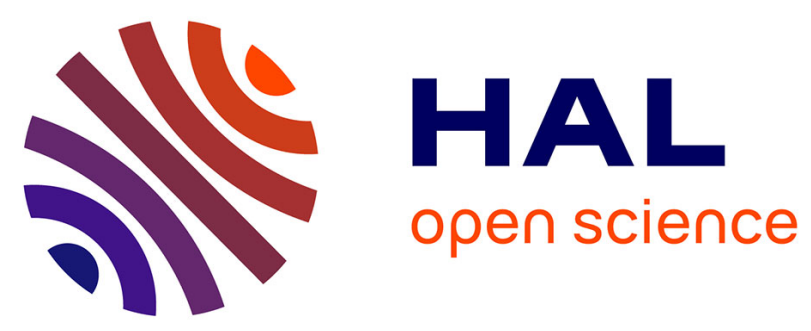

\title{
Detection of fine modifications in bulk corneal grafts based on backscattered light quantification
}

\author{
Marion Gil, Silvia Soare, Gaëlle Georges, Laure Siozade Lamoine, Matthieu \\ Gonzalvez, Louis Hoffart, Camille Guérin, Carole Gard, Gaelle Ho Wang Yin, \\ Carole Deumie
}

\section{To cite this version:}

Marion Gil, Silvia Soare, Gaëlle Georges, Laure Siozade Lamoine, Matthieu Gonzalvez, et al.. Detection of fine modifications in bulk corneal grafts based on backscattered light quantification. Optics Communications, 2019, 434, pp.246-252. 10.1016/j.optcom.2018.11.006 . hal-02415426

\author{
HAL Id: hal-02415426 \\ https://hal.science/hal-02415426
}

Submitted on 11 Mar 2020

HAL is a multi-disciplinary open access archive for the deposit and dissemination of scientific research documents, whether they are published or not. The documents may come from teaching and research institutions in France or abroad, or from public or private research centers.
L'archive ouverte pluridisciplinaire $\mathbf{H A L}$, est destinée au dépôt et à la diffusion de documents scientifiques de niveau recherche, publiés ou non, émanant des établissements d'enseignement et de recherche français ou étrangers, des laboratoires publics ou privés. 


\title{
Detection of Fine Modifications in Bulk Corneal Grafts Based on Backscattered Light Quantification
}

\author{
Marion Gila,b,*, Silvia Soare ${ }^{\mathrm{c}}$, Gaëlle Georges ${ }^{\mathrm{a}}$, Laure Siozade Lamoine ${ }^{\mathrm{a}}$, Matthieu \\ Gonzalvez $^{c}$, Louis Hoffart ${ }^{c}$, Camille Guerin ${ }^{b}$, Carole Gard ${ }^{b}$, Gaëlle Ho Wang Yin, \\ Carole Deumie ${ }^{\text {a }}$ \\ ${ }^{a}$ Aix Marseille Univ, CNRS, Centrale Marseille, Institut Fresnel, F-13013 Marseille, France \\ ${ }^{\mathrm{b}}$ Code Laboratoires Horus Pharma, 148 avenue Guynemer, Cap Var 06700 Saint-Laurent du Var, France \\ c France Service d'Ophtalmologie de l'Hôpital de la Timone, APHM, Université d'Aix-Marseille, 264 rue Saint \\ Pierre, 13385 Marseille Cedex 5, France
}

\begin{abstract}
A very sensitive technique for quantifying corneal backscattered light which measures the angleresolved light scattering throughout the reflected space is proposed. Its efficiency to detect different scattering states is compared to that of a technique currently used in hospitals: the Pentacam®. This technique, based on analyzing the backscattered light close to the specular beam, is less sensitive and it is demonstrate first on agaragar samples, for which the level of scattering is controlled. In a second time, both techniques are applied on corneal grafts and the first result is confirmed: the Pentacam $®$ detects an edema after the corneal graft has swollen by hundreds of micrometers; the proposed technique detects the change in scattering before the cornea start to swells. Since the scattering measurements are performed in the reflected space, this highly sensitive method could be applied to in-vivo analysis.
\end{abstract}

Keywords: backscattered light, cornea, sensitivity threshold, diagnostic tool.

*Marion Gil, E-mail: marion.gil@fresnel.fr

\section{Introduction}

One of the main characteristics of corneal tissue is its transparency due to low scattering and low absorption of light. This allows almost all the incident light in the visible part of the spectrum to be transmitted. Transparency depends on small variations in the refractive index of the stroma that constitutes about $90 \%$ of the cornea's thickness, coupled with the stroma layer's highly-ordered stratified structure [1] [2] [3] [4]. The stroma is composed of about 300 piledup 2- $\mu \mathrm{m}$-thick lamellas. These lamellas consist of highly-uniform collagen fibrils with a mean diameter of $31 \mathrm{~nm}$, homogeneously spaced and aligned with each other [1]. Studies on this structure revealed a phase-destructive phenomenon that interferes with the individual scattering behavior of the fibrils. This causes light to be transmitted preferably in the specular propagation direction, with very low scattering, as long as the regular fibrillar lattice is maintained at least 
in short-range order [2] [5] [6]. Above this stroma layer lies the epithelium, linked to the stroma via the Bowman's membrane and about $50 \mu \mathrm{m}$ thick. The corneal endothelium (about $5 \mu \mathrm{m}$ thick) located on the posterior of the cornea (Figure 1) regulates hydration of the cornea and maintains it at its physiological value.

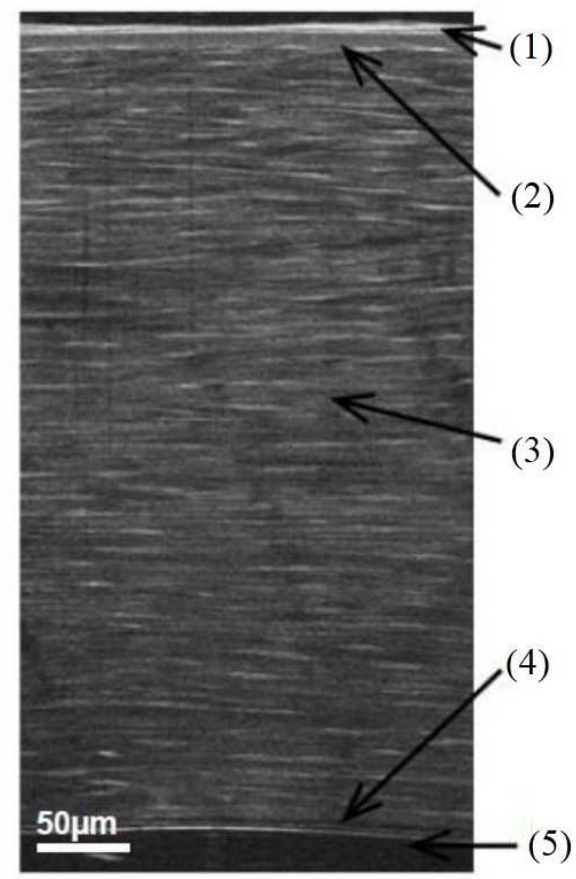

Fig. 1 Section of the human cornea measured by Full-Field Optical Coherence Tomography (1: epithelium, 2: Bowman's membrane 3: stroma, 4: Descemet membrane, 5: endothelium)

It is possible for the cornea to lose its organization, leading to increase light scattering and a consequent loss of transparency. For example, light scattering can increase after photorefractive keratectomy [7], which frequently results in perturbations to corneal organization. Scattering can also increase when an edema develops, for different reasons (following trauma, inflammation or dystrophy), the endothelial cells fail to regulate the hydration of the cornea. In pathologies leading to increase light scattering, the tissue is observed to gradually become opaque [8], which may mildly or strongly damage vision, possibly leading to blindness. It is therefore important to detect this light scattering increase early, so that it can be treated before the impact on vision becomes irreversible.

In current investigational protocols and clinical practice, to complete subjective gradation of corneal transparency because of the risk of interobserver variability and bias, quantitative 
methods of assessing corneal light scattering to qualify corneal transparency have been developed [8]-[24].

The contribution of this paper is a new technique to quantify backscattered light based on angleresolved scattered light intensity measurements. To prove the potentiality of the proposed method in comparing it to existing methods of corneal scattering quantification, a review of different techniques is first reported. They are classed into two categories, as a function of experimental result report: transmitted measurement or images. Among methods presented in the review, we choose to compare the threshold of sensitivity to a variation in the angular resolved backscattered light of the proposed method to that of the Pentacam ${ }^{\circledR}$, because it is the only hospital tool which directly propose a parameter called densitometry of the corneal backscattered light.

\section{Review of techniques for quantifying corneal transparency}

Existing methods of quantifying corneal transparency include analyzing corneal scattered light from images taken by hospital diagnostic tools. Other innovative methods are principally used on eye bank tissue.

One of this type of methods, based on hospital equipment images, is the Pentacam ${ }^{\circledR}$, a corneal topography system relying on analyses of Scheimpflug images [9]. A reference image is projected on the cornea and a Scheimpflug rotating camera measures the reflection. On each measurement, the slit of the camera is rotated about the central axis and images are captured at evenly spaced angles. The standard software provided with the instrument reconstructs the 3dimensional inner and outer surfaces of the cornea and calculates the average brightness in predefined regions of the cornea. Here, average image brightness in twelve cylindrical volumes of tissue is used. The cylinder annulus ranged between $0-2 \mathrm{~mm}, 2-6 \mathrm{~mm}, 6-10 \mathrm{~mm}$ and $10-12$ $\mathrm{mm}$ from the apex of the cornea. Each was measured at three heights: the anterior $120 \mu \mathrm{m}$, 
posterior $60 \mu \mathrm{m}$ and the central layer between these anterior and posterior regions (Figure $2 \mathrm{a}$ ). The mean image brightness, termed densitometry, is expressed as a percentage $(0-100)$ of the maximum brightness recordable by the image system. [10]. In the rest of this paper, the term densitometry denotes the average of the mean densitometry of all the different annulus (redboxed value on Figure $2 b$ ).

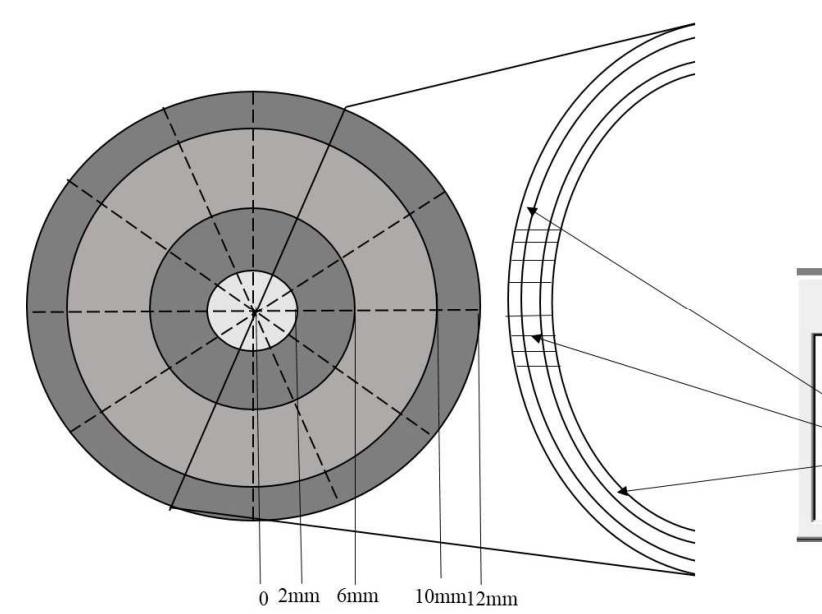

(a)

\begin{tabular}{|c|c|c|c|c|c|}
\hline \multicolumn{6}{|c|}{$\begin{array}{l}\text { Cornea densitometry } \\
\text { annulus and layer averages }\end{array}$} \\
\hline & $0 \cdot 2 \mathrm{~mm}$ & $2 \cdot 6 \mathrm{~mm}$ & 6- $10 \mathrm{~mm}$ & $10 \cdot 12 \mathrm{~mm}$ & Tatal \\
\hline Anteriar $(120 \mu m)$ & 74.1 & 65.3 & 57.3 & 56.4 & 62.8 \\
\hline Center layer & 529 & 43.0 & 41.1 & 46.0 & 44.5 \\
\hline Posterior [60 $0 \mu \mathrm{m}]$ & 43.4 & 35.9 & 32.4 & 42.8 & 36.9 \\
\hline Total & 56.8 & 48.0 & 43.6 & 48.4 & 48.0 \\
\hline
\end{tabular}

(b)

Fig 2: (a) Corneal volumes selected by the Pentacam Scheimpflug camera. (b) Mean image brightness determined in each annulus volume. The anterior annulus volume includes the anterior $120 \mu \mathrm{m}$ of cornea, the posterior includes the posterior $60 \mu \mathrm{m}$, and the central layer includes the region between these boundaries (given by the manufacturer).

It has been demonstrated that densitometry can be used to quantify backscattered light and could be used to quantify corneal transparency [11] [12].

Optical Coherence Tomography (OCT) images can also be analyzed to quantify corneal light scattering [13]. OCT is based on low-coherence interferometry and allows three-dimensional imagery. It can therefore be used to assess corneal thickness, an important parameter in diagnosing edema. If the resolution is high enough, OCT can reveal lakes of water between the lamellas [14]. In vivo studies demonstrated that analyzing the backscattered light from OCT corneal images enables corneal transparency to be quantified [15]. When the average measured 
pixel intensity of a cornea which had lost transparency was compared with that of a normal cornea, average pixel intensity was shown to vary significantly with varying corneal opacification. However, the technique is only applied to mild and severe corneal opacities [14]. Another system analyzes images from a Confocal Microscopy Through Focusing method (CMTF). CMTF is based on confocal microscopy, where the illumination and observation systems have common focal points. CMTF is currently used to observe microstructure modifications of tissue in cases of corneal degenerations or dystrophies, and to obtain endothelial cell counts [7]. With CMTF, the central cornea is scanned from the epithelium to the endothelium. The scans are initially video-recorded and later digitized. From digital images, CMTF intensity curves are generated by calculating the average pixel intensity in the central $180 \times 180$ pixel region of each image in the scan, and plotting this as a function of $\mathrm{z}$-depth. Peaks in this intensity profile are then empirically correlated to unique corneal layers [16]. Corneal backscattered light is quantified objectively by calculating the area of the CMTF peak. [17] [18] [19].

Another method consists in analyzing images taken with a slit lamp to quantify corneal scattered light and to correlate this parameter to corneal transparency [20]. The slit lamp involves an observation microscope focused on the anterior segment of the eye, generally binocular, coupled with a lighting device that projects a slit of adjustable size and orientation on the observed region. Quasi-histopathological sections of the transparent medium of the eye, and consequently of the cornea, are thus obtained. In hospital, the slit lamp is among the tools used to subjectively assess the transparency of the cornea. It has been demonstrated that analyzing the intensity of the images' pixels enables the scattered light to be quantified as an indicator of corneal transparency [21].

All the above methods are applicable in vivo. 
All the methods discussed below can only be applied ex-vivo and therefore are mainly used in research or in eye tissue banks to qualify the state of the grafts. They are based on analyze of the transmitted signal through the cornea.

One system used in eye tissue banks refers the transparency value, based on the modulation transfer function principle, as the ratio of local contrasts of a special Light Emitting Diode (LED) backlit chart measured with and without the cornea [22]. There is also systems which directly measures the light transmitted by the cornea. A light beam illuminates the center of the cornea and a sensor detects light passing through, giving the average corneal transparency [23] [24].

Other research is conducted without using the tissue bank, employing spectrophotometry to assess light transmission and explores the impact of certain pathologies or treatments [25].

The different methods presented in this section lead to real quantification of corneal transparency, principally via a quantification of corneal scattered light or a direct transmission measurement.

Unlike proposed in this paper, all are based on analyzing light reflected or transmitted by the cornea close to the specular beams. In these configurations the losses by scattering are masked by direct reflexion or transmission. Actually, any technique relying on an optical imaging system is necessarily limited by the numerical aperture. Then, it will be restricted to obtaining spatial information with spatial frequencies lower than the cutoff frequency of the imaging system. The proposed technique is based on analyzing angle-resolved scattered light measurements collected throughout the reflected space. This makes it possible to collect and analyze information relative to higher spatial frequencies $\sigma$, thereby gaining access to a smaller scale of the corneal tissue [26]. Our objective here was to prove that the angular resolved scatterometer sensitivity to a variation in backscattered light is higher than of a method that 
collects the light close to the specular directions. We chose to compare to the Pentacam® because it is the only system directly proposing a parameter, densitometry, that identifies backscattered light [27] [28].

\section{Angle-resolved scattering measurement and experimental protocol}

The interaction between light and the corneal tissue results in a complex propagation of light. One part of the incident beam is reflected and transmitted in the specular directions. However a proportion of the light is scattered through space in all directions, with an intensity depending on the angle. Surface roughness or bulk heterogeneities are responsible for light scattering [29] [30] [31]. Far-field light scattering measurement has been extensively investigated over recent decades as a non-destructive and very sensitive technique to characterize surfaces and bulks. Interpreting measurements via electromagnetic theories yields the optical properties of materials: roughness spectrum, heterogeneity index, etc [32]. However, because the level of light scattering may be low compared with the specular beam, the measurements require an efficient setup with a high dynamic range.

In the setup used in this study, a circle $5 \mathrm{~mm}$ in diameter on the sample was illuminated via a Helium Neon (HeNe) laser $(632.8 \mathrm{~nm})$. A detector was placed on a rotating arm able to perform a $180^{\circ}$ measurement of the scattered intensity. For this study, light intensity was measured for every one degree movement of the detector. A measurement at a given angle lasts approximately one second. The results presented in the rest of this paper are based on measurements of approximately 30 seconds. Figure 3 shows the scattered light intensity measurement as a function of the scattering angle $\theta$ (angle between the sample normal and the detector's position). We focused on reflected space because this is the only space that can be characterized in vivo. 
From the angle-resolved measurements, we were able to extract some integrated values overall comparison of the different samples. The Total Integrated Scattering (TIS) is defined as follows:

$$
T I S=\iint_{\theta}^{\varphi} S(\theta, \varphi) \sin (\theta) d \theta d \varphi
$$

with $(\theta, \varphi)$ the spherical coordinates of the locally measured point and $S(\theta, \varphi)=$ $B R D F \cdot \cos (\theta)$, where BRDF means Bidirectional Reflectance Distribution Function. The corneal graft is isotropic [33], so the measurements were made in the incident plane $(\varphi=0)$.

To compare to imaging systems transfer function (limited by numerical aperture), the associated optical bandwidth, in this case, is defined as $\sigma=\left[\frac{2 \pi}{\lambda} \sin \theta_{\min }, \frac{2 \pi}{\lambda} \sin \theta_{\text {max }}\right]$, with $\sigma$ the spatial frequency [8]. In general, surface scattering is observed around specular directions, with a correlation length of about $1 \mu \mathrm{m}$. By contrast, under bulk scattering, light is scattered isotropically in all directions through space, leading to a correlation length of about a fraction of a micrometer [8] [32]. Since our objective here was to study changes in scattered light due to volume modification, we needed to limit any surface effect from variations in corneal surface between samples. Differing corneal sample surfaces due to manipulation could well distort the TIS value and could hide the bulk contribution. To limit this effect, the angular range of interest needed to start at angles above $35^{\circ}$, and our experimental configuration excluded angles above $70^{\circ}$. Consequently, measurements were performed and TIS was calculated for scattering angles between $35^{\circ}$ and $70^{\circ}$. With this angular range between $35^{\circ}$ and $70^{\circ}$, we obtained an optical bandwidth in the range $[0.0057 ; 0.0093] \mathrm{nm}^{-1}$. Compared to imaging-based systems, angleresolved scattering gives access to higher spatial frequencies and consequently to a smaller scale and better resolution [8].

The dynamic range of the setup was determined by comparing two measurements, one performed without a sample (noise level), and the other with a Lambertian sample [34] used to calibrate measurements. In this setup, the dynamic range was 3 decades, large enough to 
measure precisely the level of scattering of highly-scattering or low-scattering corneas (Figure $3 b)$.

We induced different states of edema in corneal grafts in order to vary the scattering level. All the grafts used in this study were rejected by the tissue bank of Marseille due to physiological issues (low endothelial cell density, for example). The grafts were stored at ambient temperature in CorneaJet ${ }^{\circledR}$ (Eurobio, France), a deturgescence liquid. The samples were thicker than under in-vivo conditions $(600 \mu \mathrm{m}$ vs. $400 \mu \mathrm{m}$ in-vivo) [35]. The edema was induced by soaking samples for 120 minutes in a Balanced Salt Solution (BSS), a hypo osmolar solution. This lead to tissue swelling and the resulting increase in backscattered light is measured. The TIS calculated from the angular curve in the initial (thin) "low- scattering" state was denoted TIS ${ }_{0 .}$ The TIS calculated in the modified (edematous) state was denoted TIS $_{1}$ (Figure $3 b$ ). Thickness was measured via an OCT for each state (low-scattering and highly-scattering).

After swelling for 120 minutes, scattering and TIS increased.

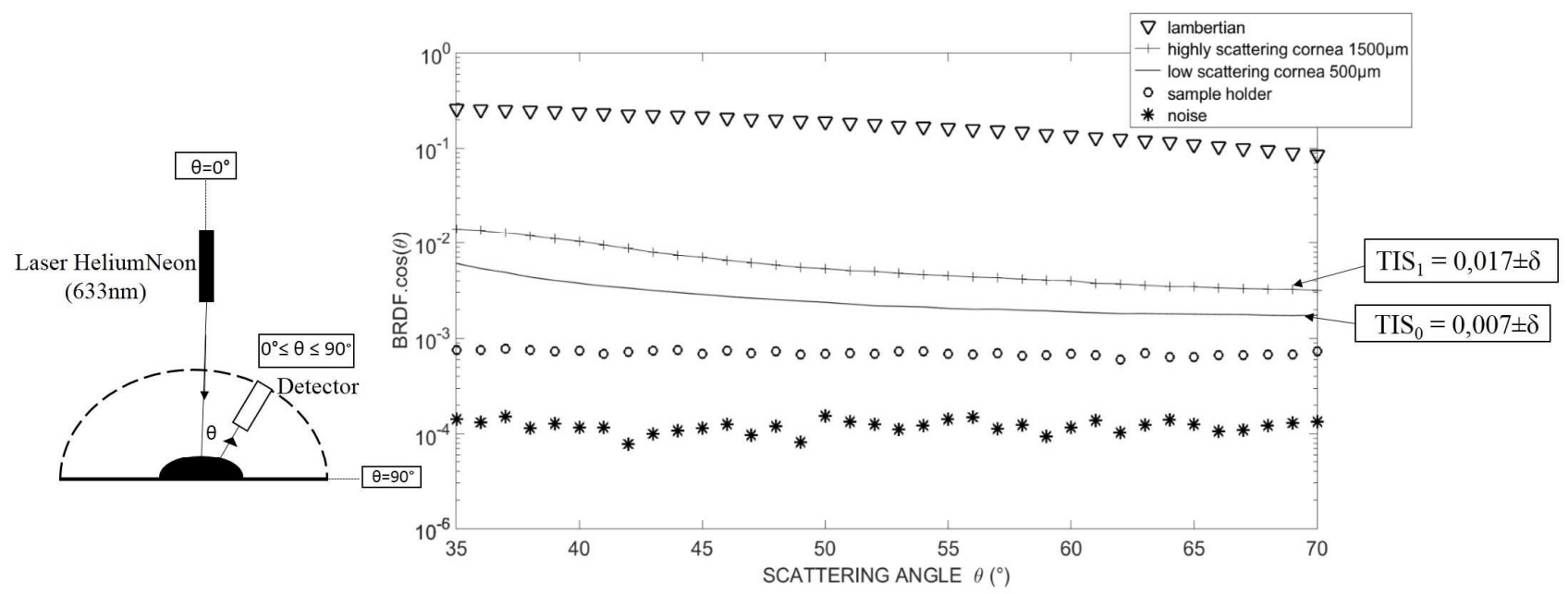

(a)

(b)

Fig. 3 (a) Angle-resolved scattering measurement principle. (b) BRDF.cos $\theta$ (with BRDF: Bidirectional Reflectance Distribution Function) of both low scattering and edematous higher scattering corneal grafts placed on a microscope slide with their associated Total Integrated Scattering (TIS) respectively TIS $_{0}$ and TIS $_{1}$ with the error $\delta=1 \%$ associated. This is compared with backscattered light of a single microscope slide. Measurements of the scattering of a Lambertian sample and of the noise are also shown so as to quantify the dynamics of the setup. The sample center was illuminated with a normal incidence. 
The performance and sensitivity to a variation on corneal scattered light of the proposed method are validated by comparison with that of the Pentacam ${ }^{\circledR}$.

\section{Repeatability and sensitivity threshold of the technique: use of agar-agar-samples}

First to quantify the performance of the experimental techniques, sample models are measured.

These models were composed of agar-agar, a substance currently used for tissue mimicking particularly in acoustics [36] [37]. The scattering levels of these samples can be controlled. In addition, all agar-agar samples were of the same thickness, $1500 \mu \mathrm{m}( \pm 10 \mu \mathrm{m})$, to ensure that only the influence of the change in scattering was being measured and assessed. So these samples are used here to determined threshold of sensitivity to a variation in scattered light, i.e. the threshold below which a change in scattered light will not be detected of both methods. A range of 27 agar-agar-models was created. Concentrations ranged from 1\%, representing a similar scattering level that of a transparent thin corneal graft of around $500 \mu \mathrm{m}$ thickness, to $3.5 \%$, representing a similar level of scattering of an edematous corneal graft of around 1500 $\mu \mathrm{m}$ thickness (Figure 4). Steps of $0.1 \%$ were used to allow fine variations in scattering.

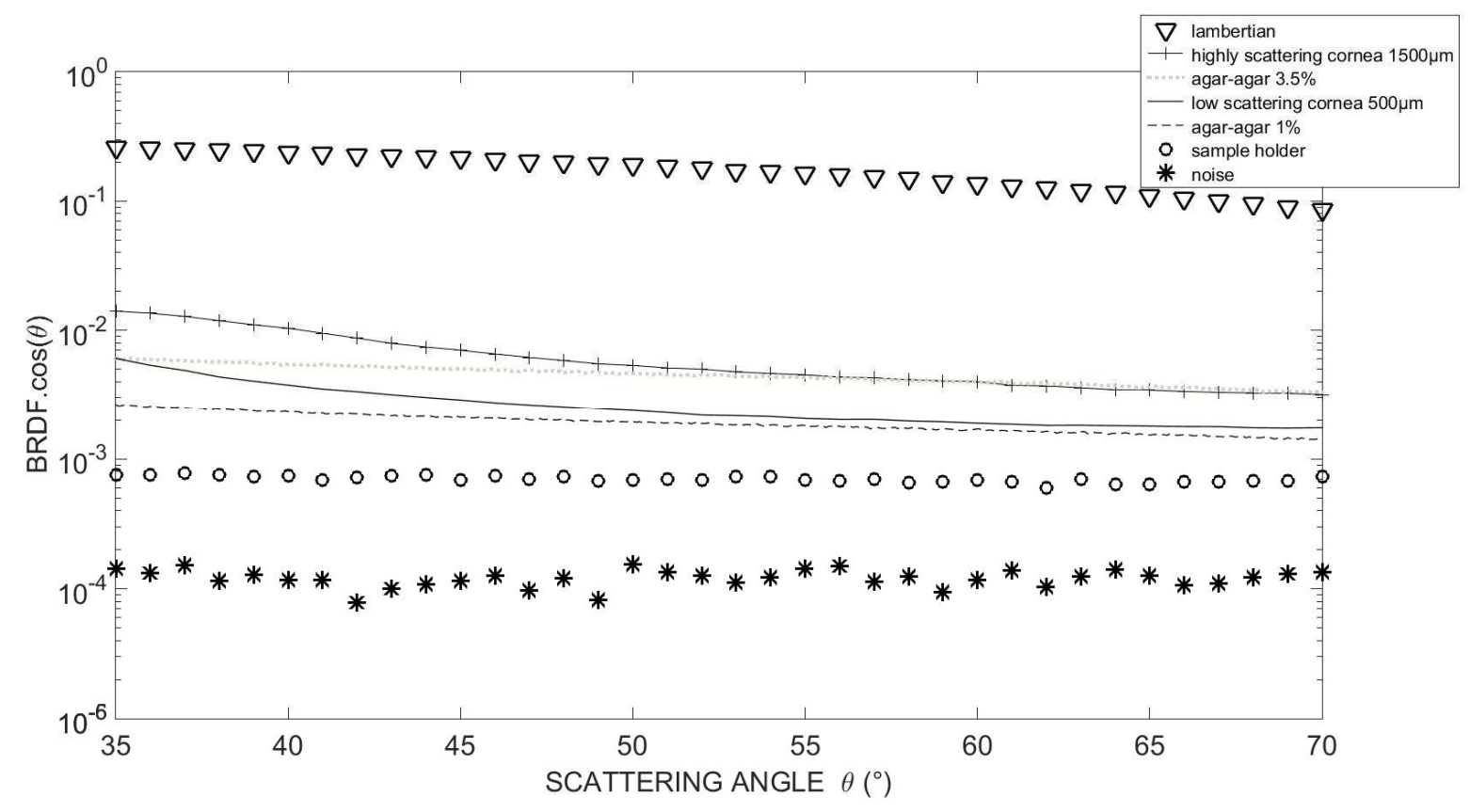


Fig. 4 Calibration of the agar-agar models. BRDF. $\cos \theta$ of both a low-scattering thin and a more highly-scattering edematous corneal graft placed on a microscope slide compared with agar-agar models of $1 \%$ and $3.5 \%$ in concentration. Measurements of a Lambertian sample, of the noise and of the sample holder are also shown so as to quantify the dynamics of the setup.

First, repeatability was assessed by analyzing each of the 27 agar-agar model samples five times in a row, for both methods. Although these samples scatter homogeneously in volume, meaning that any variation in concentration manifest as a shift of the level of scattering, we measured the backscattered light at each angle to assess angular repeatability. This would be useful in diagnosis of corneal pathologies which could present angular signature. The error associated to the measurements corresponds to the relative standard deviation calculated from a one-factor ANalyse Of Variance (ANOVA) test.

The error value was defined as the sensitivity threshold, i.e. the minimum difference required to consider two measurements as different.. The error associated to the angular resolved scatterometer is $1 \%$. For the Pentacam ${ }^{\circledR}$, the threshold was $5 \%$. The Pearson correlation coefficient between the backscattered light obtained from both methods is 0.93 .

Based on the error established for each method, their sensitivity thresholds to a variation in scattered light are compared. With the Pentacam ${ }^{\circledR}$, taking into account for the repeatability and the potential error of the measurements (represented with error bars in Figure 5), samples whose concentration is between $1 \%$ and $1.3 \%$ (Figure 5a) cannot be distinguished from one another. By contrast, when the angle-resolved scattered intensity measurement technique is used in this range, which reproduces early increased light scattering, the gap between the different angular curves is always greater than the error value previously established. Thus, with this method, all the samples between $1 \%$ and $1.3 \%$ are distinguishable from one another. 


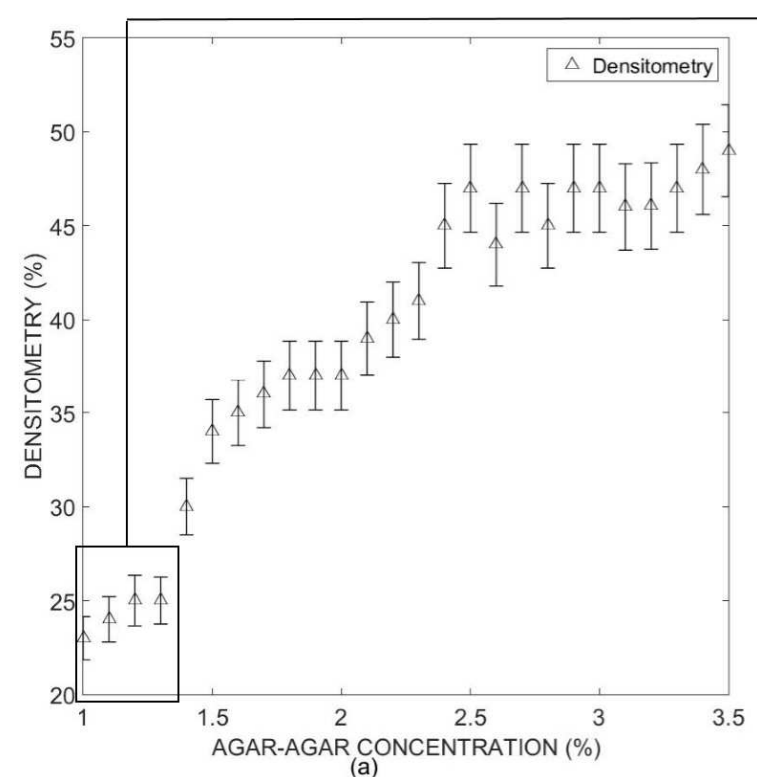

(a)

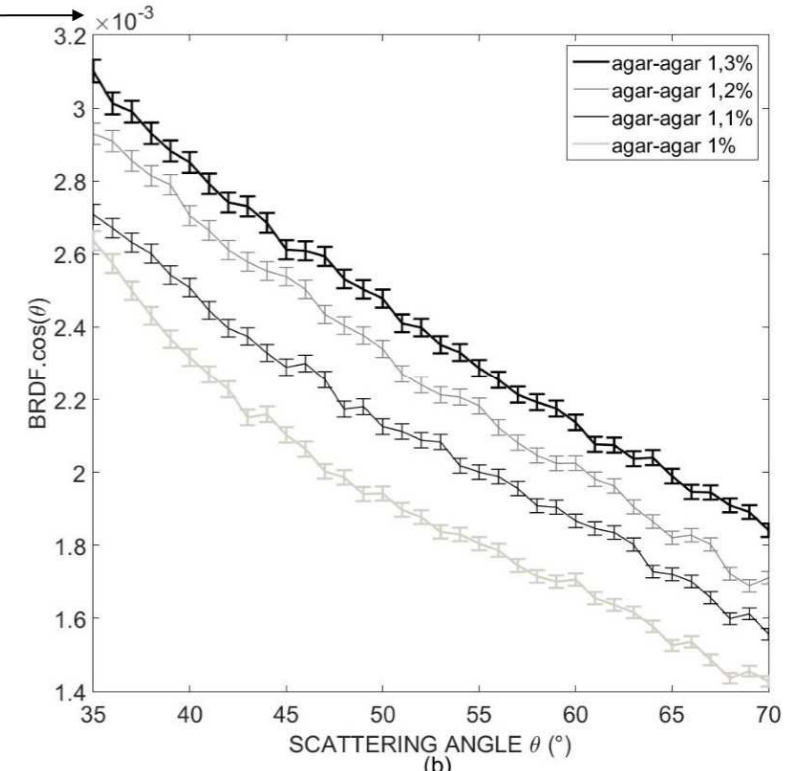

(b)

Fig. 5 (a) Densitometry (\%) according to agar-agar concentration, with error bars.

(b) Angular scattering curve for agar-agar models with a concentration between $1 \%$ and $1.3 \%$.

It can therefore be concluded that, in model samples representing controlled variations in scattered light, sensor precision and the dynamics of the angle-resolved scattered light intensity measurements provide a lower threshold of sensitivity to a variation in scattered light than that of the Pentacam ${ }^{\circledR}$. These performances are available for all the range of angle measured. This greater sensitivity of angle-resolved scattered light intensity measurements allows us to detect modifications in light scattering not visible via the Pentacam ${ }^{\circledR}$.

\section{$5 \quad$ Application to corneal grafts}

The sensitivity of this technique to detect a small variation in bulk inhomogeneity could be particularly interesting for the detection of pathologies which affect the organization of the different layers of the cornea and particularly the stroma. This last part, focus on the potentiality of this technique on corneal grafts compared to the Pentacam®. So, to assess whether this method detects variations in corneal light scattering undetectable with the Pentacam ${ }^{\circledR}$, identical protocol than with agar-agar samples was performed. First, the repeatability of both methods with corneal grafts is determined and then their sensitivity thresholds are compared. Four 
different corneal grafts with different level of scattering $\left(\right.$ TIS $_{\text {graft } 1}=0.016$, TIS $_{\text {graft } 2}=0.014$,

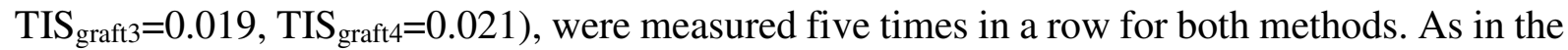
first part the relative standard deviation associated is calculated from an ANOVA test. This error value was $5 \%$ for both the angular resolved scatterometer and the Pentacam ${ }^{\circledR}$. To compare sensitivity thresholds, different levels of edema were induced in two corneal grafts, thus varying the degrees of backscattered light. Here, edemas were induced by soaking the corneal samples in BSS for 120 minutes. The two corneal grafts studied had different initial thickness, respectively $500 \mu \mathrm{m}$ for the first graft and $750 \mu \mathrm{m}$ for the second. Four times during the experiment, at the beginning (t0), after 15 minutes in BSS (t1), after 60 minutes in BSS (t2) and after 120 minutes in BSS (t3), the following were measured: (i) densitometry using Pentacam®, (ii) thickness via an OCT, and (iii) angle-resolved scattered light intensity. Unlike the scattered light variations in agar-agar samples, induced edemas led to heterogeneous variations in corneal volume (stroma) and density (lakes). Edemas do not all propagate in the same way. These modifications are not revealed by the TIS, but angular behavior provides more information on the location, size and form of heterogeneities [8].
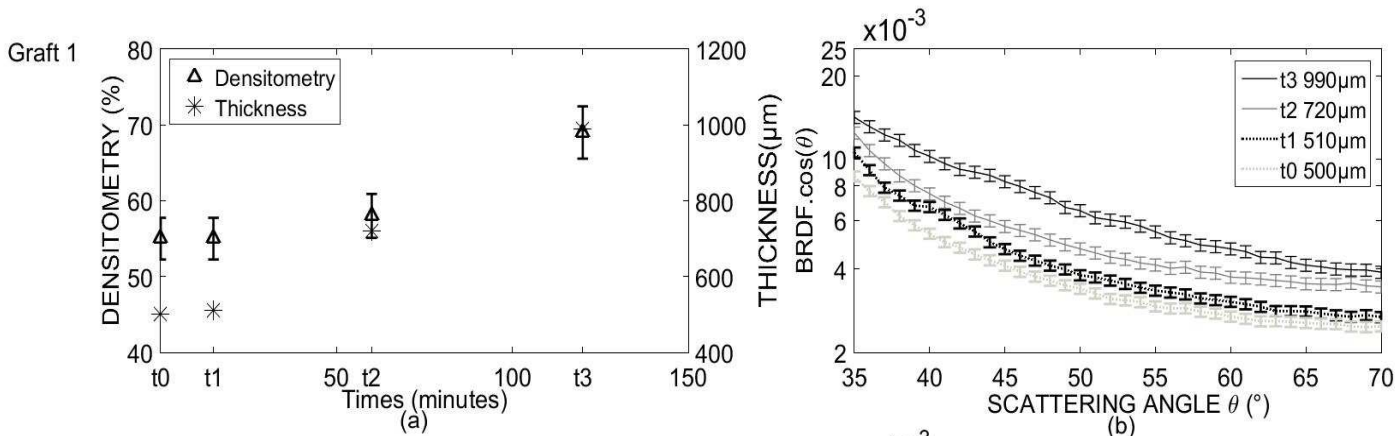

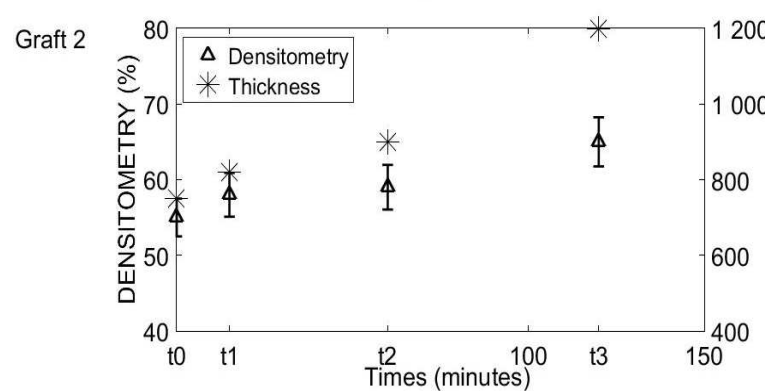

(c)

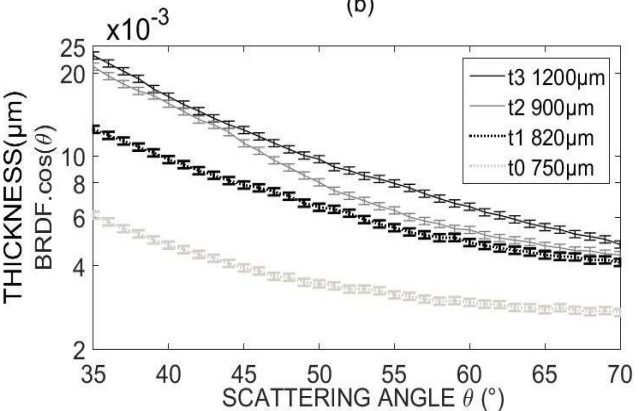

(d) 
Fig. 6 (a) Monitoring of the swelling of the first corneal graft over 120 minutes by measuring densitometry and thickness as a function of time with measurement done at the initial state, after 15, 60 and 120 minutes of swelling. (b) Angle-resolved scattered light intensity measurements of the first graft at the initial state and after 15,60 and 120 minutes of swelling. (c) Monitoring of the swelling of the second corneal graft over 120 minutes by measuring densitometry and thickness as a function of time with measurement done at the initial state, after 15, 60 and 120 minutes of swelling. (d) Angle-resolved scattered light intensity measurements of the second graft at the initial state and after 15, 60 and 120 minutes of swelling.

Results from both the Pentacam ${ }^{\circledR}$ and the angle-resolved scattered light intensity measurements (represented with error bars in Figure 6) show that for both corneal grafts, the first state where densitometry reaches a 3\% difference from the initial state is $\mathrm{t} 3$ (120 minutes of swelling). Therefore the Pentacam ${ }^{\circledR}$ does not detect backscattered light variations until there has been swelling for 120 minutes. Angle-resolved scattered light intensity measurements, however, show a gap between angular curves that is always greater than the error associated. Thus, all the different states are distinguishable from one another. This experiment therefore confirms that angle-resolved scattered light intensity measurements have a lower threshold of sensitivity to a variation in backscattered light than the Pentacam ${ }^{\circledR}$. A change in backscattered light can thus be detected earlier, making it possible to clinically diagnose edema at an earlier stage in its development using the method proposed here.

The importance of angular behavior should be noted. The different states of scattering of graft 1 are shifted as with the agar-agar sample. However, this is not the case for graft 2 where, for example, for the third state, the increase at low angles is significantly higher than at high angles. We were also able to distinguish between the grafts in terms of the type of swelling involved, on the basis of angular behavior. Here, the first, thinner graft did not swell between the first and the second states but maintained its initial thickness, whereas the second graft showed swelling, with a considerable increase in scattering, between the two states. It can thus be conjectured that there were already lakes of water in the graft [8]. But despite such a lake of water in one of the corneal grafts, Pentacam ${ }^{\circledR}$ still did not distinguish between the two samples in the second state. 


\section{Conclusion}

This paper outlined a method to qualify the cornea state based on measuring angular-resolved backscattered light. This technique differs from other backscattered quantification methods in that it does not collect the light close to the specular directions. The resulting measurement is correlated to the backscattered light measurement obtained with the Pentacam®. But, the resulting quantification is more precise and offers a lower sensitivity threshold than the Pentacam ${ }^{\circledR}$. The proposed method distinguishes between samples with levels of scattering too similar to be distinguished with the Pentacam ${ }^{\circledR}$. Applied to the cornea, it enables also earlier diagnosis of a variation in backscattered light than the Pentacam®. Our findings indicate that this method provides an innovative tool for ophthalmologists in the management of corneal dystrophies where corneal scattering is impaired. Moreover, given the sensitivity, the repeatability and the fact that the measurements are done in the reflected space this method could be applied in vivo and ophthalmologists may be able to establish an earlier diagnosis of the pathology.

\section{Disclosures}

The authors declare that they have no conflict of interest.

This research did not receive any specific grant from funding agencies in the public, commercial, or not-for-profit sectors

\section{Acknowledgments}

The project leading to this publication received funding from Excellence Initiative of AixMarseille University - A*MIDEX, a French "Investissements d'Avenir" programme

The authors thank Dr. Nouaille de Gorce and the tissue bank service of the Etablissement Français du Sang de Marseille for providing the grafts necessary for the study. 
The authors thank the platform CERIMED, "Centre Europeen de Recherche en Imagerie MEDicale" for their collaboration. We are grateful to Marjorie Sweetko for English language revision.

Reference

[1] D.Maurice, "The structure and transparency of the cornea," J. Physiol 36, pp. 263-286, 1957.

[2] G. Benedek, "Theory of transparency of the eye," Applied Optics 10(3), 1971.

[3] J.L. Cox, R.A.Farrell, R.W. Hart, M.E. Langham, "The transparency of the mammalian cornea," J. Physiol. 210, pp. 601-616, 1970.

[4] R.L. McCally, R.A. Farrell, Principles and Practice of ophtalmology, Philidelphia: W.B Saunders, 2000.

[5] J. Nishida, Cornea: Anatomy and physiology, 2nd edition, Elsevier, 2005.

[6] R.A. Farrell, R. McCally, "On corneal transparency and its loss with swelling," Applied Physics Laboratory 66(4), 1976.

[7] C. P. Lohmann, G.T. Timberlake, F.W. Fitzke, D.S. Gartry, M.K. Muir, J. Marshall, "Corneal light scattering after excimer laser photorefractive keratectomy: the objective measurement of haze.," Refrative and corneal surgery, pp. 8:114-121, 1992.

[8] G. Georges. L. S. Lamoine. C. Deumié. L. Hoffart. O. Casadessus, "Light scattering from edematous human corneal grafts' microstructure: experimental study and electromagnetic modelization," Biomedical Optics Express, vol. 3, no. 8, 2012.

[9] A. Wegener, "Photography of an anterior eye segment according to Scheimpflug's principle: options and limitations a review," Clinical and experimental Ophtalmology, pp. 37: 144-154, 2009.

[10] J. W. McLaren, K. Wacker, K.M. Kane, S.V. Pantel, "Measuring Corneal Haze by Using Scheimpflug Photography and Confocal Microscopy," Invest Ophtalmol Vis Sci, pp. 57:227-235, 2016.

[11] C. Van de Pol, K. Soya, D.G. Hwang, "Objective assessment of transient corneal haze and its relation to visual performance after photorefractive keratectomy," Am. J. Ophtalmol, pp. 132-204, 2001.

[12] A.Takacs, K. Mihaltz, Z.Z. Nagy, "Corneal density with pentacam after photorefractive keratectomy," J Refract Surg, pp. 27: 269-277, 2011.

[13] J.H. Wuang, T. Simpson, D. Fonn, "Objective measurements of corneal light backscatter during corneal swelling, by optical coherence tomography," Invinstigative Ophtalmology \& visual science, pp. Vol 45, no 10, 2004.

[14] G. Latour, G. Georges, L. Siozade Lamoine, C. Deumié-Raviol, J. Conrath, L. Hoffart,"human graft cornea and laser incisions imaging with micrometer scale resolution full field optical coherence tomography," Journal of Biomedical Optics, p. 15(5) 056006, 2010.

[15] J.S. Rose, J. Eldrina, A. Joshua, S. Amalan, T. Sebastian, S. Solomon, S. Korah, "Objective quantification of corneal haziness using anterior segment optical coherence tomography," Journal of Current Ophtalmology, 2017. 
[16] HF. Li, W. Mattheew, T. Moller-Pedersen, J.K.Maurer, H.D. Cavanagh, J.V. Jester, "Epithelial and corneal thickness measurements by in vivo confocal microscopy through focusing (CMTF)," Cur eye Res, 1997.

[17] T. Moller-Pedersen, H.D. Cavanagh, W.M. Petroll, J.V. Jester, "Stromal wound healing explains refractive instability and haze development after photorefractive keratectomy," American Academy of Ophtalmology, vol. 107, no. 7, pp. 1235-1245, 2000.

[18] T. Moller-Pedersen, M. Vogel, H.F. Li, W.M. Petroll, H.D. Cavanagh, J.V. Jester, "Quantification of stromal thinning epithelial thickness, and corneal haze after photorefractive keratectomy using in vivo confocal microscopy," Ophtalmology, vol. 104, pp. 360-368, 1997.

[19] T. Moller-Pedersen, H.D. Cavanagh, W.M. Petroll, J.V. Jester, "Corneal haze development after PRK is regulated by volume of stromal tissue removal," Cornea, vol. 17, pp. 627-639, 1998.

[20] R.E. Braustein, S.Jain, RL. McCally, WJ. Stark, P.J. Connolly, D.T.Azar, "Objective measurement of corneal light scattering after excimer laser keratectomy," Ophtalmology, vol. 103, no. 3, pp. 439-443, 1996.

[21] M.J. Maldonado, V. Arnau, A.Navea, R.Martinez-Costa, F.M. Mico, A.L. Cisneros, J.L. Menezo, "Direct Objective Quantification of corneal haze after excimer laser photorefractive keratectomy for high myopia," Ophtalmology, vol. 103, pp. 1970-1978, 1996.

[22] S. Acquart, N. Campolmi, Z. He, G. Pataia, R. Jullienne, O. Garraud, F. Nguyen, M. Péoc'h, T. Lépine, G. Thuer, P. Gain,"Non invasive measurement of transparency, arcus sensilis, scleral rim diameter of corneas during eye banking," Celll Tissue Bank, p. 15: 471:482, 2014.

[23] L. Ventura, G. Torres de Jesus, G.C. Dablas de Oliveira, S. JF. Sousa "Portable light transmission measuring system for preserved corneas," BioMedical Engineering OnLine, p. 4:70, 2005.

[24] M. Parekh, S. Ferrari, A. Ruzza, M. Pugliese, D. Ponzin, G. Salvalaio, "A portable device for measuring donor corneal transparency in eye banks," Cell Tissue Bank, pp. 15:7-13, 2014.

[25] C. Cejka, T. Ardan, J. Sirc, J. Michalek,, B. Brunova, J. Cejkova, "The influence of various toxic effects on the cornea and changes in corneal light transmission," Grafes Arch Clin Exp Ophtalmol, pp. 248: 1749-1756, 2010.

[26] C. Amra, D. Torricini, P. Roche, "Multiwavelenght (0.45-10.6 $\mu \mathrm{m})$ angle resolved scatterometer or how to extend the optical window," Applied Optics, pp. 32(28): 54625474, 1993.

[27] S.N’1 Dhubhghaill, J.J.Rozema, S.Jongenelen, I.R.Hidalgo, N.Zakaria, M.J. Tassignon, "Normative Values for Corneal Densitometry Analysis by Scheimpflug Optical Assessment," Invest Ophtalmol Vi Sc, no. 55, pp. 162-168, 2014.

[28] K.Wacker, K.M. Kane, J.W. McLaren, "Measuring Corneal Haze by Using Scheimpflug Photography and Confocal Microscopy," invest Ophtalmol Vis Sci, no. 57, pp. 227-235, 2015.

[29] C.Amra, C. Grèzes-Besset, L. Burel "Comparison of surface and bulk scattering in optical multilayers," Appl. Opt. , vol. 32, no. 28, 1993.

[30] C. Amra, "Light scattering from multilayer optics. I. Tools of investigation," Journal of the Optical Society of America A, vol. 11, pp. 197-210, 1994. 
[31] C. Deumié, R. Richier, P. Dumas, C.Amra, "Multiscale roughness in optical multilayers: atomic force microscopy and light scattering," Applieds Optics, vol. 35, pp. 5583-5594, 1996.

[32] G. Latour, G. Georges, L. Siozade-Lamoine, C.Deumié, J. Conrath, L. Hoffart, "Light scattering from human corneal grafts: Bulk and surface contribution," Journal of Applied physics, vol. 108, no. 053104, 2010.

[33] S. Mar, M.C. Martinez Garcia, T. Blanco-Mezquita, R.M. Torres, "Measurment of correlation between transmission and scattering during wound healing in hen corneas," Journal of Modern optics, vol. 56, no. 8, pp. 1014-1021, 2009.

[34] C. Amra, "Light scattering from multilayer optics. II.Application to experiment," Journal of the Optical Society of America, vol. 11, pp. 211-226, 1994.

[35] E. Pels, "Eye bank tissues: preservation technique: warm versus cold storage," Int. Ophtalmol, vol. 28, no. 3, pp. 155-163, 2008.

[36] C. Damianou. G. Menikou, "Acoustic and thermal characterization of agar based phantoms used for evaluating focused ultrasound exposures," Journal of therapeutic ultrasound, vol. 5, no. 14, 2017.

[37] A. Jozefczak, K. Kaczmarek, M. Kubovcikova, Z. Rozynek, T. Hornowski, "The effect of magnetic nanoparticles on the acoustic properties of tissue-mimicking agar-gel phantoms," Journal of Magnetism and Magnetic Materials, vol. 431, pp. 172-175, 2017.

\section{Figures}

Fig. 1 Section of the human cornea measured by Full-Field Optical Coherence Tomography (1: epithelium, 2: Bowman's membrane 3: stroma, 4: Descemet membrane, 5: endothelium)

Fig 2: (a) Corneal volumes selected by the Pentacam Scheimpflug camera. (b) Mean image brightness determined in each annulus volume. The anterior annulus volume includes the anterior $120 \mu \mathrm{m}$ of cornea, the posterior includes the posterior $60 \mu \mathrm{m}$, and the central layer includes the region between these boundaries (given by the manufacturer).

Fig. 3 (a) Angle-resolved scattering measurement principle. (b) BRDF.cos $\theta$ (with BRDF: Bidirectional Reflectance Distribution Function) of both low scattering and edematous higher scattering corneal grafts placed on a microscope slide with their associated Total Integrated Scattering (TIS) respectively TIS $_{0}$ and TIS $_{1}$ with the error $\delta=2.10^{-8}$ associated. This is compared with backscattered light of a single microscope slide. Measurements of the scattering of a Lambertian sample and of the noise are also shown so as to quantify the dynamics of the setup. The sample center was illuminated with a normal incidence.

Fig. 4 Calibration of the agar-agar models. BRDF. $\cos \theta$ of both a low-scattering thin and a more highly-scattering edematous corneal graft placed on a microscope slide compared with agar-agar models of $1 \%$ and $3.5 \%$ in concentration. Measurements of a Lambertian sample, of the noise and of the sample holder are also shown so as to quantify the dynamics of the setup.

Fig. 5 (a) Densitometry (\%) according to agar-agar concentration, with error bars.

(b) Angular scattering curve for agar-agar models with a concentration between $1 \%$ and $1.4 \%$. 
Fig. 6 (a) Monitoring of the swelling of the first corneal graft over 120 minutes by measuring densitometry and thickness as a function of time with measurement done at the initial state, after 15, 60 and 120 minutes of swelling. (b) Angle-resolved scattered light intensity measurements of the first graft at the initial state and after 15,60 and 120 minutes of swelling. (c) Monitoring of the swelling of the second corneal graft over 120 minutes by measuring densitometry and thickness as a function of time with measurement done at the initial state, after 15, 60 and 120 minutes of swelling. (d) Angle-resolved scattered light intensity measurements of the second graft at the initial state and after 15, 60 and 120 minutes of swelling. 